\title{
Doubly heavy baryon spectra guided by lattice QCD
}

\author{
H. Garcilazo,,$* *$ A. Valcarce, ${ }^{2,+}$ and J. Vijande ${ }^{3,+}$ \\ ${ }^{1}$ Escuela Superior de Física y Matemáticas, \\ Instituto Politécnico Nacional, Edificio 9, 07738 México D.F., Mexico \\ ${ }^{2}$ Departamento de Física Fundamental e IUFFyM, \\ Universidad de Salamanca, E-37008 Salamanca, Spain \\ ${ }^{3}$ Departamento de Física Atómica, Molecular y Nuclear, \\ Universidad de Valencia (UV) and IFIC (UV-CSIC), E-46100 Valencia, Spain
}

(Dated: Version of July 1, 2021)

\begin{abstract}
This paper provides results for the ground state and excited spectra of three-flavored doubly heavy baryons, bcn and bcs. We take advantage of the spin-independent interaction recently obtained to reconcile the lattice $\mathrm{SU}(3)$ QCD static potential and the results of nonperturbative lattice QCD for the triply heavy baryon spectra. We show that the spin-dependent potential might be constrained on the basis of nonperturbative lattice QCD results for the spin splittings of threeflavored doubly heavy baryons. Our results may also represent a challenge for future lattice QCD work, because a smaller lattice error could help in distinguishing between different prescriptions for the spin-dependent part of the interaction. Thus, by comparing with the reported baryon spectra obtained with parameters estimated from lattice QCD, one can challenge the precision of lattice calculations. The present work supports a coherent description of singly, doubly and triply heavy baryons with the same Cornell-like interacting potential. The possible experimental measurement of these states at $\mathrm{LHCb}$ is an incentive for this study.
\end{abstract}

PACS numbers: 14.40.Lb,12.39.Pn,12.40.-y

\footnotetext{
*Electronic address: humberto@esfm.ipn.mx

${ }^{\dagger}$ Electronic address: valcarce@usal.es

${ }^{\ddagger}$ Electronic address: javier.vijande@uv.es
} 


\section{INTRODUCTION}

In a recent publication [1] we have pointed out that the static three-quark potential with parameters determined from $\mathrm{SU}(3)$ lattice QCD [2] does not reproduce the triply heavybaryon $b b b$ and $c c c$ spectra measured also in lattice QCD [3] 5]. We argued several possible reasons for such disagreement. In a subsequent work [6] we demonstrated that the spectra of baryons containing three identical heavy quarks, $b$ or $c$, could be reproduced by means of a Cornell-like interaction, a simple Coulomb plus linear confining potential. As it happens in the heavy meson spectra, a larger value of the Coulomb strength than predicted by $\mathrm{SU}(3)$ lattice QCD was concluded. The phenomenological strengths of the Coulomb potential reproducing the heavy meson, $a$, and the triply-heavy baryon spectra, $A$, was found to satisfy $A / a<1 / 2$, slightly different from the $1 / 2$ rule as the one-gluon exchange result [7]. The strength concluded for the linear confining interaction was also slightly larger than the results from SU(3) lattice QCD. The spectra obtained in Ref. [6] supported a coherent description of the $b b b$ and $c c c$ heavy baryon spectra with the same Coulomb and confining strengths in a constituent quark model approach.

In Ref. [6] it was also pointed out that the description of the $c c c$ spectra is improved with the additional contribution of a spin-spin term, because relativistic effects are more important than in the $b b b$ case and spin-dependent contributions start playing a significant role. Although the spin-spin interaction comes suppressed by $M_{Q}^{-2}$, it helped to correctly allocate the $\operatorname{ccc}$ negative parity excitations with respect to the radial excitations of the $J^{P}=3 / 2^{+}$ground state. However, due to the identity of the three quarks, in a $Q Q Q$ system there cannot exist a good diquark, a couple of quarks with total spin 0 in a relative $S$ wave, where the spin-spin term is attractive and its contribution becomes relevant.

Thus, being the spin-independent part of the quark-quark interaction constrained by the triply heavy baryon spectra, it remains to analyze the spin-dependent part of the quarkquark potential. The best testing ground for this purpose are baryons made of three distinguishable quarks, with one of them light. As compared to singly heavy baryons, they are free of the uncertainties of the interaction between light quarks [8], whose spin-dependent part would be dominant, and the light quark kinematics [9]. Unlike triply heavy baryons, there exist pairs of quarks with total spin 0 in a relative $S$ wave, whose contribution will become crucial to study the spin splittings recently reported by nonperturbative lattice 
QCD $[10[12]$.

In this work we aim to analyze doubly heavy baryons with non-identical heavy quarks within a constituent quark model framework by means of a simple Cornell-like potential guided by lattice QCD. The spin-independent part of the quark-quark interaction has been determined from the triply heavy baryon spectra recently calculated by means of nonperturbative lattice QCD techniques and inspired by the static potentials derived within SU(3) lattice QCD. The spin-dependent part will be analyzed in comparison with the recent spinsplitting results derived in nonperturbative lattice QCD [10 12]. We will show how the spin-dependent part might be constrained on the basis of nonperturbative lattice QCD results for three-flavored doubly heavy baryons, bcn and bcs. Analogously, we will emphasize the importance of having lattice QCD results with smaller lattice errors, what could help in distinguishing between different prescriptions for the spin-dependent part of the interaction. For our purposes, we will present an exact calculation solving the Faddeev equations for three non-identical particles.

The road we outline in this work is similar to the path went through to study the heavy meson spectra. Once charmonium and bottomonium spectra were understood within a constituent quark model framework by means of simple Cornell-like potentials [13, 14], the question of predicting and trying to understand the structure of open-flavor mesons with a heavy-quark was soon posed [15]. Compared to the heavy meson case we have the great advantage of the guidance of nonperturbative lattice QCD results and the static potentials derived within SU(3) lattice QCD, which combined with the exact method to solve the threebody problem, makes the difference between our work and other studies of doubly heavy baryons [16 29]. Our results may also serve for a future analysis of the validity of the socalled superflavor symmetry, relating the spectra and properties of singly heavy mesons and doubly heavy baryons [30, 31], broken by the smallness of the heavy quark masses, which makes the size of the heavy diquark not small enough compared to $1 / \Lambda_{Q C D}$. Additional symmetries including excitations of the heavy diquark [32] could also be tested against our results.

A substantial basis for optimism in the observation of bcn ( $n$ stands for a light $u$ or $d$ quark) and bcs doubly heavy baryons is the large number of doubly heavy mesons $B_{c}(b \bar{c})$ measured at the $\mathrm{LHCb}$ [33], indicating that simultaneous production of $b \bar{b}$ and $c \bar{c}$ pairs which are close to each other in space and in rapidity and can coalesce to form doubly heavy 
hadrons is not too rare. The cross section of pair doubly heavy diquark $(b c)$ production in high energy proton-proton collisions has been already estimated [34]. It has also been recently discussed the production of doubly heavy flavored hadrons in $e^{+} e^{-}$colliders [35] as well as the doubly heavy baryon photoproduction in the future $e^{+} e^{-}$International Linear Collider (ILC) within the framework of non-relativistic QCD [36].

The paper is organized as follows. In the next section we will briefly review the parametrization of Cornell potential we have determined to get a unified description of the nonperturbative lattice QCD $b b b$ and $c c c$ spectra. We will use Sec. III to discuss the solution of the non-relativistic Faddeev equations for three non-identical particles. In Sec. IV] we will present and discuss the results of our work. Finally, in Sec. $\mathrm{V}$, we will summarize the main conclusions of this study.

\section{A POTENTIAL MODEL FOR DOUBLY HEAVY BARYONS}

The spin-independent part of the quark-quark interaction in a baryon should be the analog of the famous Cornell potential for quarkonium. The short-distance behavior is expected to be described by the two-body Coulomb potential as the one-gluon exchange (OGE) result in perturbative QCD. It should be extended for the baryon case, with a factor $1 / 2$ in front of its strength due to color factors [7]. As for the $Q \bar{Q}$ case, the characteristic signature of the long-range non-Abelian dynamics is believed to be a linear rising of the static interaction. Moreover, the general expectation for the baryonic case is that, at least classically, the strings meet at the so-called Fermat (or Torricelli) point, which has minimum distance from the three sources ( $Y$-shape configuration) [37, 38]. The confining short-range potential could be also described as the sum of two-body potentials $(\Delta$-shape or linear configuration) [37-40]. We have shown in Ref. [6] the equivalence of both prescriptions for the case of triply heavy baryons (see Table II of that reference) for different values of the heavy-quark mass. Thus, a minimal model to study doubly heavy baryons comes given by,

$$
V\left(r_{i j}\right)=-A \sum_{i<j} \frac{1}{\left|\vec{r}_{i}-\vec{r}_{j}\right|}+B \sum_{i<j}\left|\vec{r}_{i}-\vec{r}_{j}\right| .
$$

The value of the $Q \bar{Q}$ confinement strength, $b$, is usually fixed to reproduce that obtained from the linear Regge trajectories of the pseudoscalar $\pi$ and $K$ mesons, $\sqrt{\sigma}=(429 \pm 2)$

$\mathrm{MeV}$ [41]. In the case of baryons, the linear string tension $B$ is considered to be of the 
order of a factor $1 / 2$ of the $Q \bar{Q}$ case. The reduction factor in the string tension can be naturally understood as a geometrical factor rather than a color factor, due to the ratio between the minimal distance joining three quarks and the perimeter length of a triangle, suggesting $B=(0.50 \sim 0.58) b[2]$. For the particular case of quarks in an equilateral triangle $B=\frac{1}{\sqrt{3}} b=0.58 b$ [38]. When the linear ansatz is adopted for the two-body potential, still the same relation holds for the strength of the Coulomb potential $A \simeq \frac{1}{2} a$, due to color factors. The $\Delta$ ansatz (linear potential) has been widely adopted in the nonrelativistic quark model because of its simplicity [8, 42-47]

On the other hand, potential models are also less accurate for baryons containing light quarks, because relativistic effects are more important and spin-dependent contributions may start playing a significant role. Although of small importance in heavy quark systems for being suppressed as $M_{Q}^{-2}$, the spin-spin interaction derived from the one-gluon exchange helps to improve the description of the nonperturbative lattice QCD results [6]. Thus, an spin-spin term must be considered in the interacting potential for those systems where spindependent corrections may play a role, having the quark-quark interaction the final form,

$$
V_{S}\left(r_{i j}\right)=-A \sum_{i<j} \frac{1}{\left|\vec{r}_{i}-\vec{r}_{j}\right|}+B \sum_{i<j}\left|\vec{r}_{i}-\vec{r}_{j}\right|+A \sum_{i<j} \frac{1}{M_{i} M_{j}} \frac{e^{-r / r_{0}}}{r r_{0}^{2}}\left(\vec{\sigma}_{i} \cdot \vec{\sigma}_{j}\right) .
$$

The spin-spin interaction arising from the one-gluon exchange potential has the same strength as the Coulomb term [7]. Its $\delta(r)$ radial structure has to be regularized in order to avoid an unbound spectrum [48]. The determination of the strength of the spin-dependent part in heavy baryons with three-identical quarks is not efficient. As mentioned above, the identity of the quarks doe not allow for the existence of a good diquark, a couple of particles with total spin 0 in a relative $S$ wave, where the spin-spin term is attractive and its contribution significant. This is why doubly heavy baryons with non-identical heavy quarks are ideal systems to test the spin-dependent part of the quark-quark potential. On one hand, the problem is free of the uncertainties of chiral symmetry breaking effects related to pairs of light quarks and, on the other hand, the distinguishability of the quarks allows for the existence of all pairs in a relative $S$ wave with spin 0 .

Finally, to make contact with our previous studies of the heavy baryon spectra [8, 9], the linear potential is screened at long distances,

$$
V_{S S}\left(r_{i j}\right)=-A \sum_{i<j} \frac{1}{\left|\vec{r}_{i}-\vec{r}_{j}\right|}+B^{\prime} \sum_{i<j}\left(1-e^{-\mu\left|\vec{r}_{i}-\vec{r}_{j}\right|}\right)+A \sum_{i<j} \frac{1}{M_{i} M_{j}} \frac{e^{-r / r_{0}}}{r r_{0}^{2}}\left(\vec{\sigma}_{i} \cdot \vec{\sigma}_{j}\right)
$$


such that the same linear strength is guaranteed at short-range, $B=B^{\prime} \mu^{1}$.

\section{FADDEEV EQUATIONS FOR THREE NON-IDENTICAL PARTICLES}

After partial-wave decomposition, the Faddeev equations are integral equations in two continuous variables as shown in Ref. [50]. They can be transformed into integral equations in a single continuous variable by expanding the two-body $t$-matrices in terms of Legendre polynomials as shown in Eqs. (32)-(36) of Ref. [51]. One obtains the final set of equations,

$$
\psi_{i ; L S T}^{n \ell_{i} \lambda_{i} S_{i} T_{i}}\left(q_{i}\right)=\sum_{j \neq i} \sum_{m \ell_{j} \lambda_{j} S_{j} T_{j}} \int_{0}^{\infty} q_{j}^{2} d q_{j} K_{i j ; L S T}^{n \ell_{i} \lambda_{i} S_{i} T_{i} m \ell_{j} \lambda_{j} S_{j} T_{j}}\left(q_{i}, q_{j} ; E\right) \psi_{j ; L S T}^{m \ell_{j} \lambda_{j} S_{j} T_{j}}\left(q_{j}\right),
$$

with

$$
\begin{aligned}
K_{i j ; L S T}^{n \ell_{i} \lambda_{i} S_{i} T_{i} m \ell_{j} \lambda_{j} S_{j} T_{j}}\left(q_{i}, q_{j} ; E\right)= & \frac{1}{2}<S_{i} T_{i} \mid S_{j} T_{j}>_{S T} \sum_{r} \tau_{i ; n r}^{\ell_{i} S_{i} T_{i}}\left(E-q_{i}^{2} / 2 \nu_{i}\right) \\
& \times \int_{-1}^{1} d \cos \theta \frac{P_{r}\left(x_{i}^{\prime}\right) P_{m}\left(x_{j}\right)}{E-p_{j}^{2} / 2 \eta_{j}-q_{j}^{2} / 2 \nu_{j}} A_{L}^{\ell_{i} \lambda_{i} \ell_{j} \lambda_{j}}\left(p_{i}^{\prime} q_{i} p_{j} q_{j}\right) .
\end{aligned}
$$

$P_{r}\left(x_{i}^{\prime}\right)$ and $P_{m}\left(x_{j}\right)$ are Legendre polynomials, $x_{i}^{\prime}=\left(p_{i}^{\prime}-b\right) /\left(p_{i}^{\prime}+b\right), x_{j}=\left(p_{j}-b\right) /\left(p_{j}+b\right)$, and $b$ a scale parameter. $\tau_{i ; n r}^{\ell_{i} S_{i} T_{i}}\left(E-q_{i}^{2} / 2 \nu_{i}\right)$ are the coefficients of the expansion of the two-body $t$-matrices in terms of Legendre polynomials defined by Eq. (34) of Ref. [51]. $S_{i}$ and $T_{i}$ are the spin and isospin of the pair $j k$ while $S$ and $T$ are the total spin and isospin. $\ell_{i}$ is the orbital angular momentum of the pair $j k, \lambda_{i}$ is the orbital angular momentum of particle $i$ with respect to the pair $j k$, and $L$ is the total orbital angular momentum.

$$
\begin{aligned}
\eta_{i} & =\frac{m_{j} m_{k}}{m_{j}+m_{k}}, \\
\nu_{i} & =\frac{m_{i}\left(m_{j}+m_{k}\right)}{m_{i}+m_{j}+m_{k}},
\end{aligned}
$$

are the usual reduced masses. For a given set of values of $L S T$ the integral equations (4) couple the amplitudes of the different configurations $\left\{\ell_{i} \lambda_{i} S_{i} T_{i}\right\}$. The spin-isospin recoupling coefficients $<S_{i} T_{i} \mid S_{j} T_{j}>_{S T}$ are given by,

$$
\begin{aligned}
<S_{i} T_{i} \mid S_{j} T_{j}>_{S T}= & (-)^{S_{j}+\sigma_{j}-S} \sqrt{\left(2 S_{i}+1\right)\left(2 S_{j}+1\right)} W\left(\sigma_{j} \sigma_{k} S \sigma_{i} ; S_{i} S_{j}\right) \\
& \times(-)^{T_{j}+\tau_{j}-T} \sqrt{\left(2 T_{i}+1\right)\left(2 T_{j}+1\right)} W\left(\tau_{j} \tau_{k} T \tau_{i} ; T_{i} T_{j}\right),
\end{aligned}
$$

\footnotetext{
${ }^{1}$ As shown in Ref. [49] for the light baryon spectra, the long distance screening would just provide with a better understanding of low-spin highly excited baryons and high-spin baryons, with no significant effect on the states studied on this work.
} 
with $\sigma_{i}$ and $\tau_{i}$ the spin and isospin of particle $i$, and $W$ is the Racah coefficient. The orbital angular momentum recoupling coefficients $A_{L}^{\ell_{i} \lambda_{i} \ell_{j} \lambda_{j}}\left(p_{i}^{\prime} q_{i} p_{j} q_{j}\right)$ are given by

$$
\begin{array}{r}
A_{L}^{\ell_{i} \lambda_{i} \ell_{j} \lambda_{j}}\left(p_{i}^{\prime} q_{i} p_{j} q_{j}\right)=\frac{1}{2 L+1} \sum_{M m_{i} m_{j}} C_{m_{i}, M-m_{i}, M}^{\ell_{i} \lambda_{i} L} C_{m_{j}, M-m_{j}, M}^{\ell_{j} \lambda_{j} L} \Gamma_{\ell_{i} m_{i}} \Gamma_{\lambda_{i} M-m_{i}} \Gamma_{\ell_{j} m_{j}} \\
\times \Gamma_{\lambda_{j} M-m_{j}} \cos \left[-M\left(\vec{q}_{j}, \vec{q}_{i}\right)-m_{i}\left(\vec{q}_{i}, \vec{p}_{i}^{\prime}\right)+m_{j}\left(\vec{q}_{j}, \vec{p}_{j}\right)\right],
\end{array}
$$

with $\Gamma_{\ell m}=0$ if $\ell-m$ is odd and

$$
\Gamma_{\ell m}=\frac{(-)^{(\ell+m) / 2} \sqrt{(2 \ell+1)(\ell+m) !(\ell-m) !}}{2^{\ell}((\ell+m) / 2) !((\ell-m) / 2) !},
$$

if $\ell-m$ is even. The angles $\left(\vec{q}_{j}, \vec{q}_{i}\right),\left(\vec{q}_{i}, \vec{p}_{i}^{\prime}\right)$, and $\left(\vec{q}_{j}, \vec{p}_{j}\right)$ can be obtained in terms of the magnitudes of the momenta by using the relations

$$
\begin{aligned}
& \vec{p}_{i}^{\prime}=-\vec{q}_{j}-\frac{\eta_{i}}{m_{k}} \vec{q}_{i}, \\
& \vec{p}_{j}=\vec{q}_{i}+\frac{\eta_{j}}{m_{k}} \vec{q}_{j}
\end{aligned}
$$

where $i j$ is a cyclic pair. The magnitude of the momenta $p_{i}^{\prime}$ and $p_{j}$, on the other hand, are obtained in terms of $q_{i}, q_{j}$, and $\cos \theta$ using Eqs. (10) as

$$
\begin{aligned}
& p_{i}^{\prime}=\sqrt{q_{j}^{2}+\left(\frac{\eta_{i}}{m_{k}}\right)^{2} q_{i}^{2}+\frac{2 \eta_{i}}{m_{k}} q_{i} q_{j} \cos \theta}, \\
& p_{j}=\sqrt{q_{i}^{2}+\left(\frac{\eta_{j}}{m_{k}}\right)^{2} q_{j}^{2}+\frac{2 \eta_{j}}{m_{k}} q_{i} q_{j} \cos \theta} .
\end{aligned}
$$

The integral equations (44) couple the amplitude $\psi_{i}$ to the amplitudes $\psi_{j}$ and $\psi_{k}$. When the three particles are different, by substituting the equation for $\psi_{i}$ into the corresponding equations for $\psi_{j}$ and $\psi_{k}$, one obtains at best integral equations that involve two independent amplitudes which means that in that case the numerical calculations are more time consuming. If one represents in Eq. (44) the integration over $d q_{j}$ by a numerical quadrature [52], then for a given set of the conserved quantum numbers $L, S$, and $T$, Eq. (4) can be written in the matrix form

$$
\psi_{i}=\sum_{j \neq i} B_{i j}(E) \psi_{j},
$$

where $\psi_{i}$ is a vector whose elements correspond to the values of the indices $n, \ell_{i}, \lambda_{i}, S_{i}, T_{i}$, and $r$, i.e.,

$$
\psi_{i} \equiv \psi_{i ; L S T}^{n \ell_{i} \lambda_{i} S_{i} T_{i}}\left(q_{r}\right)
$$


with $q_{r}$ the abscissas of the integration quadrature. The matrix $B_{i j}(E)$ is given by,

$$
B_{i j}(E) \equiv q_{s}^{2} w_{s} K_{i j ; L S T}^{n \ell_{i} \lambda_{i} S_{i} T_{i} m \ell_{j} \lambda_{j} S_{j} T_{j}}\left(q_{r}, q_{s} ; E\right)
$$

where the vertical direction is defined by the values of the indices $n, \ell_{i}, \lambda_{i}, S_{i}, T_{i}$, and $r$ while the horizontal direction is defined by the values of the indices $m, \ell_{j}, \lambda_{j}, S_{j}, T_{j}$, and $s$. $q_{s}$ and $w_{s}$ are the abscissas and weights of the integration quadrature.

Substituting Eq. (12) for $i=1$ into the corresponding equations for $i=2$ and $i=3$ one obtains,

$$
\begin{aligned}
& {\left[B_{21}(E) B_{12}(E)-1\right] \psi_{2}+\left[B_{21}(E) B_{13}(E)+B_{23}(E)\right] \psi_{3}=0} \\
& {\left[B_{31}(E) B_{12}(E)+B_{32}(E)\right] \psi_{2}+\left[B_{31}(E) B_{13}(E)-1\right] \psi_{3}=0}
\end{aligned}
$$

so that the binding energies of the system are the zeroes of the Fredholm determinant

$$
|M(E)|=0
$$

where

$$
M(E)=\left(\begin{array}{cc}
B_{21}(E) B_{12}(E)-1 & B_{21}(E) B_{13}(E)+B_{23}(E) \\
B_{31}(E) B_{12}(E)+B_{32}(E) & B_{31}(E) B_{13}(E)-1
\end{array}\right)
$$

\section{RESULTS AND DISCUSSION}

To obtain the predictions of the Cornell-like potential of Eq. (3) for the bcn and bcs baryon spectra to compare with the results measured in nonperturbative lattice QCD [10 12], we solve the three-body problem for non-identical quarks by means of the Faddeev method described in Sec. III. We solve the nonrelativistic Schrödinger equation

$$
\left\{H_{0}+V(r)\right\} \Psi(\vec{r})=E \Psi(\vec{r})
$$

where $H_{0}$ is the free part of quarks without center-of-mass-motion

$$
H_{0}=\sum_{i=1}^{3}\left(m_{i}+\frac{\vec{p}_{i}^{2}}{2 m_{i}}\right)-T_{C M}
$$

and $m_{i}$ is the mass of quark $i$. The mass of the heavy baryon will be finally given by $M_{B}=m_{1}+m_{2}+m_{3}+E$. The quarks masses are taken as in Ref. [8]: $m_{b}=5.034 \mathrm{GeV}$, $m_{c}=1.659 \mathrm{GeV}, m_{s}=0.545 \mathrm{GeV}$, and $m_{u}=m_{d}=0.313 \mathrm{GeV}$, as well as the long-distance screening parameter $\mu=0.7 \mathrm{fm}^{-1}$. 
TABLE I: Faddeev amplitudes, $\left(\ell_{i}, \lambda_{i}, S_{i}, T_{i}\right)$, used in the calculation of the different $J^{P}$ states. $(L, S)$ indicates the channel giving the lowest energy, $L$ is the total orbital angular momentum and $S$ is the total intrinsic spin of the three quarks. $\ell_{i}$ is the orbital angular momentum of the pair $j k$ and $\lambda_{i}$ is the orbital angular momentum of particle $i$ with respect to the pair $j k . S_{i}$ and $T_{i}$ are indicated at the bottom of the table. For spin 1/2 each Faddeev amplitude appears twice, with $S_{i}=0$ and $1 . T_{i}$ is uniquely determined, either 0 or $1 / 2$.

\begin{tabular}{|c|cc|}
\hline$J^{P}$ & $(L, S)$ & $\left(\ell_{i}, \lambda_{i}\right)$ \\
\hline $1 / 2^{+}$ & $(0,1 / 2)^{a}$ & $(0,0),(1,1),(2,2),(3,3),(4,4),(5,5)$ \\
$3 / 2^{+}$ & $(0,3 / 2)^{b}$ & $(0,0),(1,1),(2,2),(3,3),(4,4),(5,5)$ \\
$5 / 2^{+}$ & $(2,1 / 2)^{a}$ & $(0,2),(2,0),(1,1),(1,3),(3,1),(2,2),(2,3),(3,2),(3,3)$ \\
$7 / 2^{+}$ & $(2,3 / 2)^{b}$ & $(0,2),(2,0),(1,1),(1,3),(3,1),(2,2),(2,3),(3,2),(3,3)$ \\
$1 / 2^{-}$ & $(1,1 / 2)^{a}$ & $(1,0),(0,1),(1,2),(2,1),(2,3),(3,2),(3,4),(4,3),(4,5),(5,4)$ \\
$3 / 2^{-}$ & $(1,1 / 2)^{a}$ & $(1,0),(0,1),(1,2),(2,1),(2,3),(3,2),(3,4),(4,3),(4,5),(5,4)$ \\
$5 / 2^{-}$ & $(1,3 / 2)^{b}$ & $(1,0),(0,1),(1,2),(2,1),(2,3),(3,2),(3,4),(4,3),(4,5),(5,4)$ \\
\hline
\end{tabular}

${ }^{a}$ Each Faddeev amplitude may exist with $S_{i}=0$ and 1 for the pair $j k . T_{i}$ is fixed, either 0 or $1 / 2$.

${ }^{b}$ Each Faddeev amplitude is only compatible with $S_{i}=1$ for the pair $j k . T_{i}$ is fixed, either 0 or $1 / 2$

We show in Table \ the Faddeev amplitudes that we consider to solve the three-body problem for each $J^{P}$ state, indicating the $(L, S)$ channel giving the lowest energy. As indicated in the table, for those cases with intrinsic spin 1/2, each Faddeev amplitude would contribute twice, with the two possible spin couplings of the $j k$ pair, 0 and 1 . The isospin of the pair $j k$ is fixed.

Before proceeding to analyze the results, we present in Table II the convergence of our calculation with respect to the number of the Faddeev amplitudes considered in our calculation, indicated in Table II. As we can see the results are fully converged with three Faddeev amplitudes (times its degeneracy, an additional factor two for spin $1 / 2$ and another factor two for negative parity states because they are reached with non-identical pairs of $\ell_{i}$ and $\lambda_{i}$, see Table I), i.e., when all Faddeev amplitudes with $\ell_{i}=\lambda_{i} \leq 2$ have been considered. For the sake of completeness, our results have been obtained with all amplitudes quoted in Table I, which guarantees full convergence. 
TABLE II: Convergence of the binding energy $E$ of different $J^{P}$ bcs baryons, in $\mathrm{MeV}$, with respect to the number, $N$, of Faddeev configurations $\left(\ell_{i}, \lambda_{i}\right)$. See text for details.

\begin{tabular}{|cc|cc|cc|lc|}
\hline$N$ & $E_{3 / 2^{+}}$ & $N$ & $E_{1 / 2^{-}}$ & $N$ & $E_{1 / 2^{+}}$ & $N$ & $E_{\left(1 / 2^{+}\right)^{*}}$ \\
\hline 1 & 1005 & 4 & 1226 & 2 & 953 & 2 & 987 \\
2 & 996 & 8 & 1203 & 4 & 937 & 4 & 978 \\
3 & 995 & 12 & 1199 & 6 & 934 & 6 & 977 \\
4 & 995 & 16 & 1197 & 8 & 933 & 8 & 977 \\
5 & 995 & 20 & 1196 & 10 & 932 & 10 & 976 \\
6 & 995 & & & 12 & 932 & 12 & 976 \\
\hline
\end{tabular}

We present in Fig. 1 the excitation spectra of bcs baryons with the potential of Eq. (3) and the parameters used in Ref. [6] to reproduce the nonperturbative lattice QCD results of triply heavy baryons $b b b$ and $c c c: A=0.1875, B=0.1374 \mathrm{GeV}^{2}$, and an almost constant regularization, $r_{0}$, for the spin-spin term following the line of the model AL1 in Ref. [45]. The spin-independent part of the quark-quark interaction fixes in a unique manner the position of the radial and orbital excitations. As explained above, these systems provide with an additional advantage, as compared to triply heavy baryons, that they allow to scrutinize the strength of the spin-spin interaction. In Ref. [6] it was shown how the addition of the spindependent part of the quark-quark interaction, maintaining the strength of the Coulomb and the confining potential determined from the $b b b$ spectra, allows for a better agreement in the $c c c$ case. The idea behind this improvement is that potential models probably are also less accurate for $c c c$ than for $b b b$ baryons, because the $c c c$ system is more relativistic and spin-dependent contributions may start playing a significant role.

In a three-quark baryon, any pair of quarks must be in a $\overline{\mathbf{3}}$ color state to couple to a color singlet with the $\mathbf{3}$ color state of the other quark. For identical particles, the $\overline{\mathbf{3}}$ color state is antisymmetric. Thus for a symmetric relative $S$ wave between the quarks, $\ell_{i}=0$, if they are identical they can only exist in a symmetric spin state, $S_{i}=1$. In other words, in a $Q Q Q$ triply heavy baryon there are no good diquarks, where the spin-dependent part of the interaction is attractive and significant. A pair of identical quarks could only exist in an antisymmetric spin state, $S_{i}=0$, with a unit of orbital angular momentum, which 


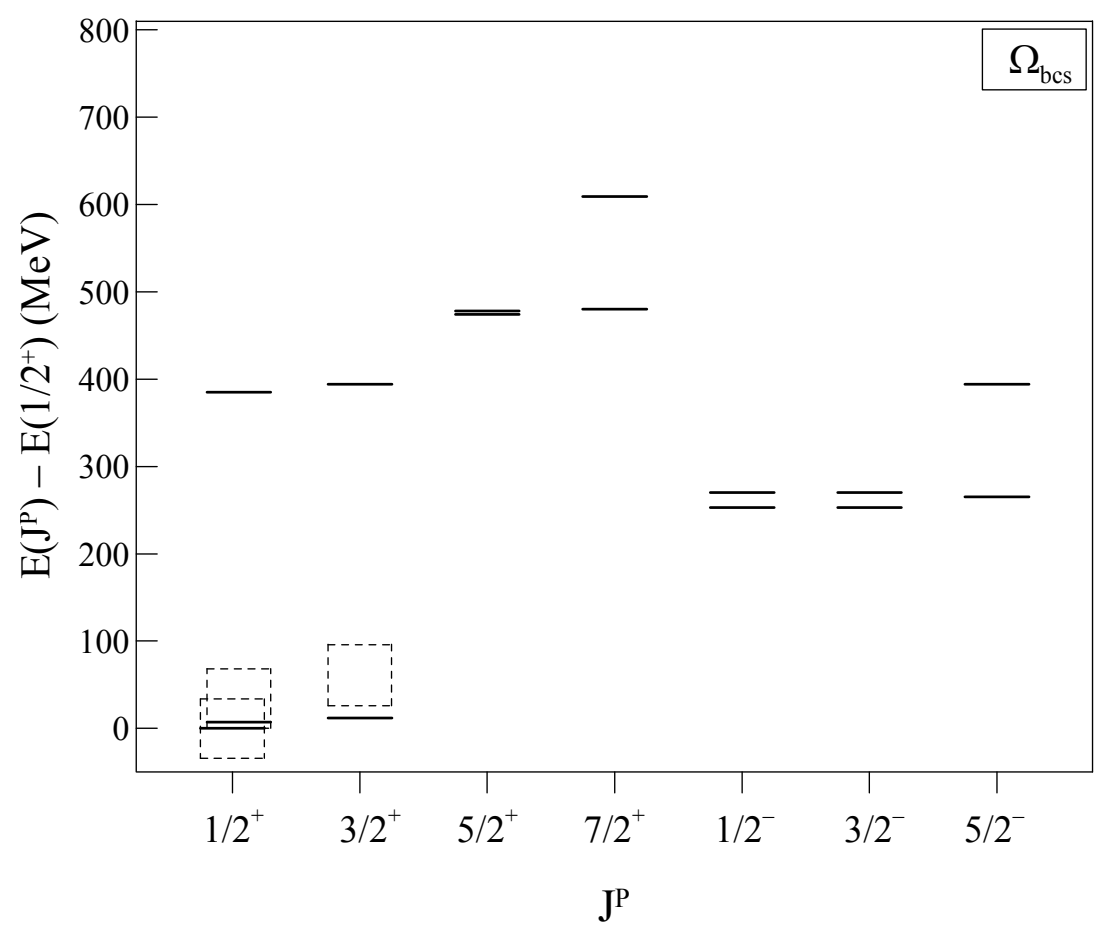

FIG. 1: bcs excited state spectra, solid lines, for the potential of Eq. (3) including a spin-spin interaction regularized as in Ref. [45]. The boxes stand for the nonperturbative lattice QCD results of Ref. [10]. See text for details.

reconciles the symmetry of the state with the Pauli principle. As the spin-spin interaction is very short-ranged, the relative $P$ wave shields its effect. The benefit of this term in the case of triply heavy baryons can be simply understood. In the ground state there are no pairs of quarks with spin zero, while there are in negative parity states. The attraction induced by the spin-spin term allows to relax the value of the quark masses diminishing the repulsive effect of the centrifugal barrier. This was the main effect observed in Ref. [6].

By using an almost constant regularization for the spin-spin interaction, as suggested by the model AL1 of Ref. [45], it is obtained a spin-splitting that it is small as compared to the central value recently obtained by nonperturbative lattice QCD [10]: $E_{\Omega_{b c}^{\prime}}-E_{\Omega_{b c}}=35 \pm 9 \pm 25$ $\mathrm{MeV}$, and $E_{\Omega_{b c}^{*}}-E_{\Omega_{b c}}=62 \pm 9 \pm 25 \mathrm{MeV}^{2}$. The predictions obtained for these spin-splittings in Fig. 1 are 7 and $12 \mathrm{MeV}$, respectively. Let us however note that these results are within

\footnotetext{
${ }^{2} \Omega_{b c}$ stands for a bcs $J^{P}=1 / 2^{+}$state with two quarks in a spin 0 state, the ground state; $\Omega_{b c}^{\prime}$ stands for a $b c s J^{P}=1 / 2^{+}$state with two quarks in a spin 1 state; $\Omega_{b c}^{*}$ stands for a $b c s J^{P}=3 / 2^{+}$state and thus any two quark pair is in a spin 1 state. The same notation is valid for $b c n$ states that are denoted by $\Xi_{b c}$.
} 
TABLE III: $E_{\Omega_{b c}^{*}}-E_{\Omega_{b c}}$ spin splitting, in $\mathrm{MeV}$, for different prescriptions of the regularization of the spin-spin interaction, $r_{0}$. See text for details.

\begin{tabular}{|c|ccc|c|}
\hline$E_{\Omega_{b c}^{*}}-E_{\Omega_{b c}}$ & $r_{0_{(c, s)}}$ & $r_{0_{(b, s)}}$ & $r_{0_{(b, c)}}$ & Latt. [10] \\
\hline 12 & & {$[45]$} & & \\
63 & & {$[8]$} & & \\
56 & {$[8]$} & {$[45]$} & {$[45]$} & $62 \pm 9 \pm 25$ \\
22 & {$[45]$} & {$[8]$} & {$[45]$} & \\
14 & {$[45]$} & {$[45]$} & {$[8]$} & \\
\hline
\end{tabular}

2 sigma of the lattice central values which highlights the importance of having smaller lattice errors to help in clearly distinguishing between different prescriptions for the spindependent part of the interaction. A similar situation is observed with the mass difference between the first two states with $J^{P}=5 / 2^{+}$or $J^{P}=1 / 2^{-}$, that are predicted to be almost degenerate, although in this case we have no lattice results to compare with. This possible underestimation of the spin-spin effects by prescriptions as that in Ref. [45] had already been noted in Ref. [8] in the study of singly heavy baryons in a constituent quark model approach, although, as we have mentioned in the introduction, in that case the presence of two light quarks did not allow a clear cut between the spin-independent and spin-dependent effects as in the present case, due to involved dynamics of the two light-quark subsystem.

To illustrate the relevance of the spin-dependent terms in three-flavored doubly heavy baryons, we evaluate the mass difference $E_{\Omega_{b c}^{*}}-E_{\Omega_{b c}}$ following different prescriptions for the regularization of the $\delta$ term in the spin-spin interaction. The results are shown in Table III. In the first case we use the almost constant regularization of Ref. [45] (see Eq. (2) of Ref. [45], $\left.r_{0} \in[0.26,0.37] \mathrm{fm}\right)$. In the second case we use the flavor dependent regularization of Ref. [8] (see Table 5 of Ref. [8], $\left.r_{0} \in[0.017,0.27] \mathrm{fm}\right)^{3}$. In the last three cases we identify which interaction is responsible for the spin splitting, that as could have been expected is the spin-spin interaction between the lightest flavors $(c, s)$, those between $(b, s)$ and $(b, c)$ being rather small. At the light of the results of the first and the last files of Table III one can easily understand the results of Ref. [6] regarding the spin-dependent part of the interaction,

\footnotetext{
${ }^{3}$ A similar recipe was used long-ago for a simultaneous study of the meson and baryon spectra in Ref. [53].
} 

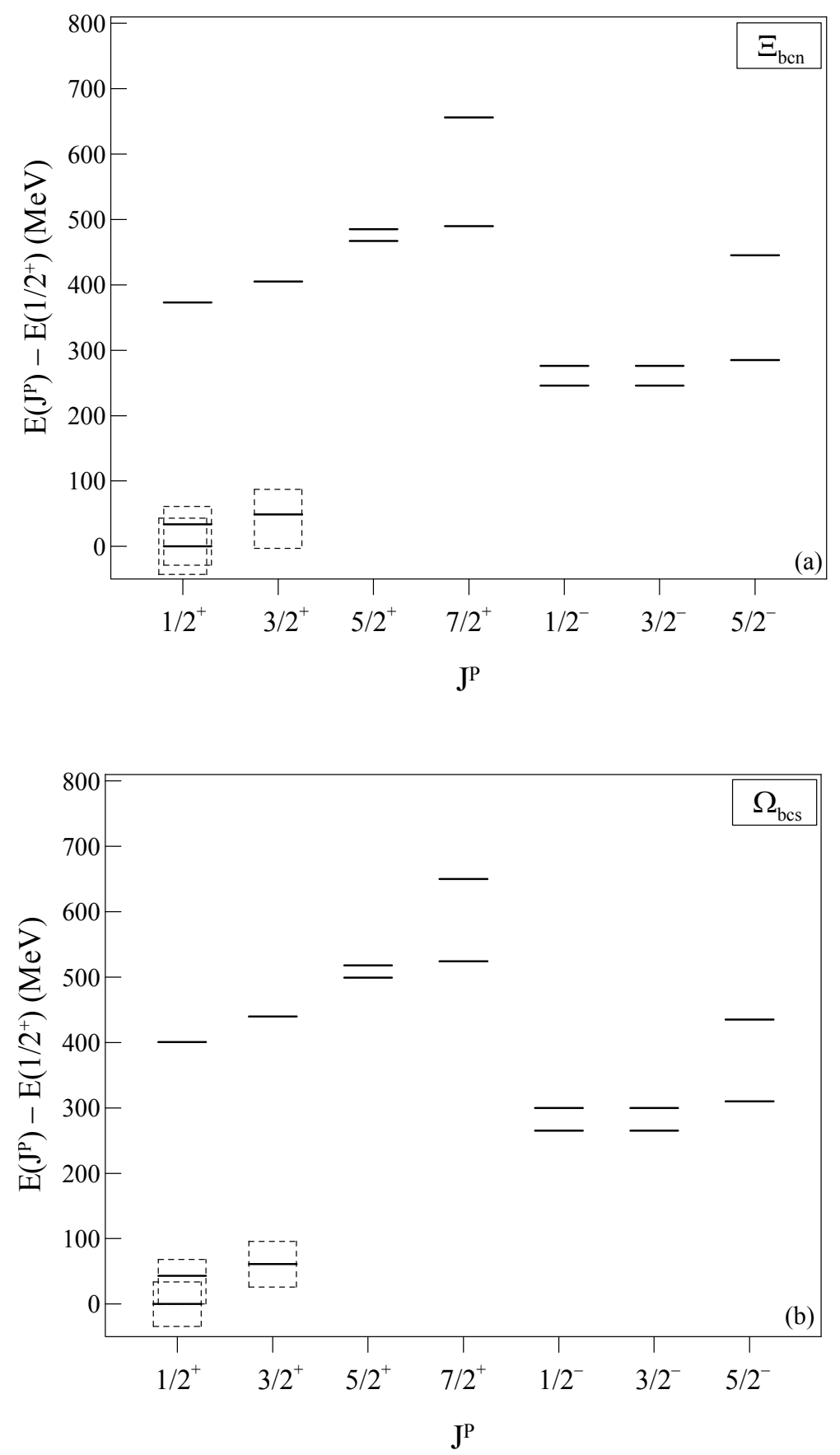

FIG. 2: (a) bcn excited state spectra, solid lines, for the potential of Eq. (3) including a spin-spin interaction regularized as in Ref. [8]. (b) Same as (a) for bcs baryons. The boxes stand for the nonperturbative lattice QCD results of Ref. [10]. See text for details.

for larger masses of the quarks the spin-splitting is almost the same independently of the prescription used for the regularization. As explained above, this is the reason why triplyheavy baryons are not adequate to analyze the spin-dependent part of the quark-quark interaction. 
Thus, using the flavor-dependent regularization of the spin-spin interaction derived in Ref. [8] to study singly heavy baryons, we have recalculated the bcs and bcn excited spectra, that are shown in Fig. 2. As mentioned above, the spin-independent part of the quarkquark interaction determined in the triply heavy baryon spectra fixes in a unique manner the position of the radial and orbital excitations, thus these states are a first challenge for future lattice works and/or experimental searches. Regarding the low-energy $J^{P}=1 / 2^{+}$ and $J^{P}=3 / 2^{+}$states, the results in Fig. 2 are close to the central values of nonperturbative lattice QCD for the spin splitting between them [10-12]. Thus, the present results can be considered as a useful challenge for future lattice QCD work because one can see how a smaller lattice error could clearly help in distinguishing between the different prescriptions for the spin-dependent part of the interaction.

Let us note the parameter-free nature of our calculation, making use of the spinindependent interaction derived in Ref. [6] from the analysis of triply heavy baryons together with the parametrization of the spin-dependent term obtained in Ref. [8] from the analysis of singly heavy baryons. Our results unify the heavy-quark dynamics for the description of triply [6], doubly and singly [8] heavy baryons by means of a simple Cornell potential with a flavor-dependent spin-spin regularization. They are therefore a nice testbench for future works of lattice QCD on the ground and excited spectra of three-flavored doubly heavy baryons. They might be useful in future projects of lattice QCD calculations and also as a guideline in future experiments looking for doubly heavy baryons with non-identical heavy quarks.

\section{SUMMARY}

In brief, the spectra of three-flavored doubly heavy baryons have been calculated by means of a Faddeev approach. The spin-independent part of the quark-quark interaction was taken for grant from a recent study of nonperturbative lattice QCD results for triply heavy baryons. As in the case of the heavy meson spectra, a larger value of the Coulomb strength than predicted by $\mathrm{SU}(3)$ lattice QCD is needed. The phenomenological strengths of the Coulomb potential reproducing the heavy meson and the triply heavy baryon spectra satisfy $A / a<1 / 2$, slightly different from the $1 / 2$ rule as the one-gluon exchange result. It has been shown that the spin-dependent part of the interaction could be fixed by studying 
the spin splitting of three-flavored doubly-heavy baryons. The adequacy of these systems to determine the regularized $\delta$-type interaction has been justified, obtaining a reasonable agreement with the central values of the spin-splittings derived by nonperturbative lattice QCD with the same flavor dependent regularization already used for singly heavy baryons. Our results make evident the importance of having at our disposal nonperturbative lattice QCD results with smaller error, what would allow to clearly distinguish between different prescriptions for the spin-dependent part of the interaction. Besides, they constitute a nice testbench for future works of lattice QCD on the excited spectra of doubly heavy baryons with non-identical heavy quarks as well as experimental searches. Let us finally note that by comparing with the reported baryon spectra obtained with parameters estimated from lattice QCD, one can challenge the precision of lattice calculations.

The detailed theoretical investigation presented in our recent works about the heavy baryon spectra based on nonperturbative lattice QCD guidance, may help to improve our understanding of the interaction in many-quark systems containing heavy quarks, of interest to deepen our understanding on intriguing recent experimental results as the so-called $X Y Z$ exotic states or the LHCb pentaquark [54]. Similarly the possible advent of new experimental data [33] as well as the improvements in lattice QCD calculations of the heavy baryon spectra [10], makes the present calculation timely to scrutinize the quark-quark interaction in systems containing heavy flavors.

\section{Acknowledgments}

We thank to N. Mathur and S. Meinel for valuable information about the present status of nonperturbative lattice QCD calculations of excited heavy baryon states. This work has been partially funded by COFAA-IPN (México), by Ministerio de Educación y Ciencia and EU FEDER under Contracts No. FPA2013-47443 and FPA2015-69714-REDT, by Junta de Castilla y León under Contract No. SA041U16, and by USAL-FAPESP grant 2015/50326-5. A.V. is thankful for financial support from the Programa Propio XIII of the University of Salamanca.

[1] J. Vijande, A. Valcarce, and H. Garcilazo, Phys. Rev. D 90, 094004 (2014). 
[2] T. T. Takahashi, H. Suganuma, Y. Nemoto, and H. Matsufuru, Phys. Rev. D 65, 114509 (2002).

[3] S. Meinel, Phys. Rev. D 82, 114514 (2010).

[4] S. Meinel, Phys. Rev. D 85, 114510 (2012).

[5] M. Padmanath, R. G. Edwards, N. Mathur, and M. Peardon, Phys. Rev. D 90, 074504 (2014).

[6] J. Vijande, A. Valcarce, and H. Garcilazo, Phys. Rev. D 91, 054011 (2015).

[7] J. -M. Richard, arXiv:1205.4326.

[8] A. Valcarce, H. Garcilazo, and J. Vijande, Eur. Phys. J. A 37, 217 (2008).

[9] A. Valcarce, H. Garcilazo, and J. Vijande, Phys. Lett. B 733, 288 (2014).

[10] Z. S. Brown, W. Detmold, S. Meinel, and K. Orginos, Phys. Rev. D 90, 094507 (2014).

[11] M. Padmanath, R. G. Edwards, N. Mathur, and M. Peardon, Phys. Rev. D 91, 094502 (2015).

[12] N. Mathur (private communication).

[13] C. Quigg and J. L. Rosner, Phys. Rep. 56, 167 (1979).

[14] E. Eichten, K. Gottfried, T. Kinoshita, K. D. Lane, and T. -M. Yan, Phys. Rev. D 21, 203 (1980).

[15] S. Godfrey and N. Isgur, Phys. Rev. D 32, 189 (1985).

[16] S. Fleck and J. -M. Richard, Prog. Theor. Phys. 82, 760 (1989).

[17] V. V. Kiselev, A. K. Likhoded, O. N. Pakhomova, and V. A. Saleev, Phys. Rev. D 66, 034030 (2002).

[18] D. Ebert, R. N. Faustov, V. O. Galkin, A. P. Martynenko, and V. A. Saleev, Z. Phys. C 76, 111 (1997).

[19] S. P. Tong, Y. B. Ding, X. H. Guo, H. Y. Jin, X. Q. Li, P. N. Shen, and R. Zhang, Phys. Rev. D 62, 054024 (2000).

[20] R. Roncaglia, D. B. Lichtenberg, and E. Predazzi, Phys. Rev. D 52, 1722 (1995).

[21] J. G. Körner, M. Krämer, and D. Pirjol, Prog. Part. Nucl. Phys. 33, 787 (1994).

[22] C. Itoh, T. Minamikawa, K. Miura, and T. Watanabe, Phys. Rev. D 61, 057502 (2000).

[23] H. Kaur and M. P. Khanna, hep-ph/0005077.

[24] D. Ebert, R. N. Faustov, V. O. Galkin, and A. P. Martynenko, Phys. Rev. D 66, 014008 (2002).

[25] R. Lewis, N. Mathur, and R. M. Woloshyn, Phys. Rev. D 64, 094509 (2001).

[26] M. Karliner and S. Nussinov, JHEP 1307, 153 (2013). 
[27] T. Yoshida, E. Hiyama, A. Hosaka, M. Oka, and K. Sadato, Phys. Rev. D 92, 114029 (2015).

[28] Y. -L. Ma and M. Harada, Phys. Lett. B 754, 125 (2016).

[29] Z. Shah, K. Thakkar, and A. Kumar-Rai, arXiv:1609.03030.

[30] M. J. Savage and M. B. Wise, Phys. Lett. B 248, 177 (1990).

[31] N. Brambilla, A. Vairo, and T. Rösch, Phys. Rev. D 72, 034021 (2005).

[32] B. Eakins and W. Roberts, Int. J. Mod. Phys. A 27, 1250039 (2012).

[33] R. Aaij et al. (LHCb Collaboration), Phys. Rev. Lett. 109, 232001 (2012); Phys. Rev. Lett. 111, 181801 (2013); Phys. Rev. Lett. 113, 152003 (2014).

[34] A. Trunin, Phys. Rev. D 93, 114029 (2016).

[35] X. -C. Zheng, C. -H. Chang, and Z. Pan, Phys. Rev. D 93, 034019 (2016).

[36] G. Chen, X. -G. Wu, Z. Sun, Y. Ma, and H. -B. Fu, JHEP 1412, 018 (2014).

[37] T. T. Takahashi and H. Suganuma, Phys. Rev. D 70, 074506 (2004).

[38] V. G. Bornyakov, H. Ichie, Y. Mori, D. Pleiter, M. I. Polikarpov, G. Schierholz, T. Streuer, H. Stüben, and T. Suzuki (DIK collaboration), Phys. Rev. D 70, 054506 (2004).

[39] J. M. Cornwall, Phys. Rev. D 69, 065013 (2004).

[40] C. Alexandrou, Ph. de Forcrand, and O. Jahn, Nucl. Phys. B Proc. Suppl. 119, 667 (2003).

[41] G. S. Bali, Phys. Rep. 343, 1 (2001), and references therein.

[42] H. Garcilazo, A. Valcarce, and J. Vijande, J. Phys. G 34, 961 (2007).

[43] N. Isgur and G. Karl, Phys. Rev. D 18, 4187 (1978).

[44] M. Oka and K. Yazaki, Prog. Theor. Phys. 66, 556 (1981).

[45] B. Silvestre-Brac, Few-Body Syst. 20, 1 (1996).

[46] E. Klempt and J. -M. Richard, Rev. Mod. Phys. 82, 1095 (2010).

[47] V. Crede and W. Roberts, Rept. Prog. Phys. 76, 076301 (2013).

[48] R. K. Bhaduri, L. E. Cohler, and Y. Nogami, Phys. Rev. Lett. 44, 1369 (1980).

[49] J. Vijande, P. González, H. Garcilazo, and A. Valcarce, Phys. Rev. D 69, 074019 (2004).

[50] A. Valcarce, H. Garcilazo, and J. Vijande, Phys. Rev. C 72, 025206 (2005).

[51] T. Fernández-Caramés, A. Valcarce, H. Garcilazo, and P. González, Phys. Rev. C 73, 034004 (2006).

[52] M. Abramowitz and I. A. Stegun, Handbook of Mathematical Functions (Dover, New York, 1972).

[53] S. Ono and F. Schöberl, Phys. Lett. B 118, 419 (1982). 
[54] H. -X. Chen, W. Chen, X. Liu, and S. -L. Zhu, Phys. Rept. 639, 1 (2016). 